\title{
Identification of a Conserved Gene Signature Associated with an Exacerbated Inflammatory Environment in the Hippocampus of Aging Rats
}

\author{
Joaquín Pardo, ${ }^{1}$ Martin C. Abba, ${ }^{2}$ Ezequiel Lacunza, ${ }^{2}$ Laetitia Francelle, ${ }^{3}$ \\ Gustavo R. Morel, ${ }^{1 \dagger}$ Tiago F. Outeiro, ${ }^{3 \dagger}$ and Rodolfo G. Goya (id ${ }^{1 \dagger}$
}

\begin{abstract}
There have been a few descriptive studies in aged rodents about transcriptome changes in the hippocampus, most of them in males. Here, we assessed the age changes in spatial memory performance and hippocampal morphology in female rats and compared those changes with changes in the hippocampal transcriptome. Old rats displayed significant deficits in spatial memory. In both age groups, hole exploration frequency showed a clear peak at hole 0 (escape hole), but the amplitude of the peak was significantly higher in the young than in the old animals. In the hippocampus, there was a dramatic reduction in neurogenesis, whereas reactive microglial infiltrates revealed an inflammatory hippocampal state in the senile rats. Hippocampal RNAsequencing showed that $\mathbf{2 1 0}$ genes are differentially expressed in the senile rats, most of them being downregulated. Our RNA-Seq data showed that various genes involved in the immune response, including TYROBP, CD11b, C3, CD18, CD4, and CD74, are overexpressed in the hippocampus of aged female rats. Enrichment analysis showed that the pathways overrepresented in the senile rats matched those of an exacerbated inflammatory environment, reinforcing our morphologic findings. After correlating our results with public data of human and mouse hippocampal gene expression, we found an 11-gene signature of overexpressed genes related to inflammatory processes that was conserved across species. We conclude that age-related hippocampal deficits in female rats share commonalities between human and rodents. Interestingly, the 11-gene signature that we identified may represent a cluster of immune and regulatory genes that are deregulated in the hippocampus and possibly other brain regions during aging as well as in some neurodegenerative diseases and low-grade brain tumors. Our study
\end{abstract}

${ }^{1}$ INIBIOLP, Histology B-Pathology B, School of Medicine, UNLP, La Plata, Argentina; ${ }^{2}$ CINIBA, School of Medicine, UNLP, La Plata, Argentina; ${ }^{3}$ Department of Neurodegeneration and Restorative Research, University Medical Center Göttingen, Göttingen, Germany

Additional Supporting Information may be found in the online version of this article.

Grant sponsor: Argentine Agency for the Promotion of Science and Technology; Grant number: \#PICT11-1273 and \#PICT13-1590; Grant sponsor: the Argentine Research Council (CONICET); Grant number: PIP0597 (RGG); Grant sponsor: DFG Center for Nanoscale Microscopy and Molecular Physiology of the Brain (CNMPB) (TFO); Grant sponsor: German-Argentine (Ale-Arg) (JP); Grant sponsor: Humboldt Research Fellowship; Grant number: Humboldt ID Number 1164643.

Abbreviations: AT, acquisition trial; BM, Barnes Maze; DG, dentate gyrus; ESTIMATE, estimation of stromal and immune cells in malignant tumor using expression data; GCL, granular cell layer; GO, gene ontology analysis; MWM, Morris water maze; PT, probe trial; SGZ, subgranular zone; SR, stratum radiatum

${ }^{\dagger}$ These three authors contributed equally to this study.

*Correspondence to: Rodolfo Goya, INIBIOLP, Faculty of Medicine, UNLP, CC 455; La Plata 1900, Argentina; E-mail: goya@isis.unlp.edu.ar Accepted for publication 29 December 2016.

DOI 10.1002/hipo.22703

Published online 13 January 2017 in Wiley Online Library (wileyonlinelibrary.com). further supports neuroinflammation as a promising target to treat cognitive dysfunction in old individuals and some brain tumors. (c) 2017 Wiley Periodicals, Inc.

KEY WORDS: aging; spatial memory; hippocampal transcriptome; immune pathways; Barnes maze

\section{INTRODUCTION}

In humans and rats, aging is associated with a progressive deterioration of spatial learning and memory. These functional alterations are correlated with morphological changes in the brain, particularly in the hippocampus, a key brain region for the formation and consolidation of spatial memory (O'Keefe et al., 1976; Squire et al., 1993). Two of the most conspicuous changes associated with aging in the rat hippocampus are a sharp decline of neurogenesis in the hippocampal dentate gyrus (Morel et al., 2015) and a progressive activation of the microglia, the immune effector of inflammation and antigen recognition in the brain (Streit et al., 2004). There is clear evidence that a constellation of gene expression changes underlie hippocampal phenotype aging. Thus, gene expression studies in aging rodents have documented significant changes in hippocampal genes related to cholesterol synthesis, inflammation, transcription factors, neurogenesis, and synaptic plasticity (Blalock et al., 2003; Verbitsky et al., 2004; Burger et al., 2007, 2008; Rowe et al., 2007). While those studies revealed that aging itself is associated with the majority of gene expression changes, a smaller portion of the transcriptional differences in the hippocampus are related to changes in learning and spatial memory performance. To our knowledge, most studies assessing age-related transcription changes in the hippocampus of rodents have been performed in males. In the only study we are aware of, electrophoretic gel shift assays were used to document that the binding activity of nuclear factor kappa B, activator protein-1, peroxisome proliferator-activated receptor, and liver X receptor in the cortex and hippocampus from young (3month old) and old (18- month old) male and female 
rats, display a complex pattern of changes in their brainbinding activity, depending on the anatomical origin of the samples (cortex or hippocampus), and the sex of the animals studied (Sanguino et al., 2006). Therefore, there still is a significant information gap concerning possible sex-related differences in the impact of aging on the hippocampal transcriptome in rodents. In humans, gene expression profiles were assessed in the hippocampus, entorhinal cortex, superior-frontal gyrus, and postcentral gyrus across the lifespan of 55 cognitively intact individuals aged 20-99 years (Berchtold et al., 2008). The results revealed clear gender differences in brain aging, suggesting that the human brain undergoes sexually dimorphic changes in gene expression not only in development but also in later life. More gene expression changes were detected in males than in females across all brain regions. Therefore, it is likely that aging in rodents is also associated with sex-related differences in gene expression in the brain. Since we have characterized the impact of aging on cognitive performance and hippocampal morphology in female rats, it was of interest to correlate these changes with transcriptome changes in the hippocampus of aging female rats. To this end, we performed RNA-seq analysis of the whole female rat hippocampus and correlated our results with public databases recording agerelated changes in gene expression in the hippocampus of male rodents and humans of both sexes.

Interestingly, we identified an 11-gene signature of overexpressed genes related to inflammatory processes conserved across species, providing novel insight into aging mechanisms and age-associated disorders in the brain.

\section{METHODS}

\section{Animals}

Two-month old (young) and 28-months old female SpragueDawley rats were used. The young and old animals (8 per group) weighed $199 \pm 1$ and $267 \pm 5$ g, respectively.

Rats were housed in a temperature-controlled room $\left(22 \pm 2{ }^{\circ} \mathrm{C}\right)$ on a 12:12 h light/dark cycle. Food and water were available ad libitum. All experiments with animals were performed in accordance to the Animal Welfare Guidelines of NIH (INIBIOLP's Animal Welfare Assurance No A5647-01). The ethical acceptability of the animal protocols used here have been approved by our institutional IACUC (Protocol \# T09-01-2013).

\section{Spatial Memory Assessment}

The modified Barnes maze protocol used here has been previously documented (Morel et al., 2015). It consists of an elevated $(108 \mathrm{~cm}$ to the floor) black acrylic circular platform, $122 \mathrm{~cm}$ in diameter, containing 20 holes around the periphery. The holes are of uniform diameter $(10 \mathrm{~cm})$ and appearance, but only one hole is connected to a black escape box (tunnel). The escape box is $38.7 \mathrm{~cm}$ long $\times 12.1 \mathrm{~cm}$ wide $\times 14.2 \mathrm{~cm}$ in depth and it is removable. A white cylindrical starting chamber (an opaque, $25 \mathrm{~cm}$ in diameter and $20 \mathrm{~cm}$ high, openended chamber) is used to place the rats on the platform with a random orientation of their bodies.

Four proximal visual cues are placed in the room, $50 \mathrm{~cm}$ away from the circular platform. The escape hole is numbered as Hole 0 for graphical normalized representation purposes, the remaining holes being numbered 1-10 clockwise, and -1 to -9 counterclockwise. During the whole experiment, Hole 0 remained in a fixed position, relative to the cues in order to avoid randomization of the relative position of the escape box. A $90-\mathrm{dB}$ white-noise generator and a white-light $500-\mathrm{W}$ bulb provided the escape stimulus from the platform. At the beginning of the experiment, rats were habituated to the task. The habituation routine consists of placing the animals in every compartment during $180 \mathrm{~s}$ (starting chamber and escape box). An acquisition trial (AT) consists of placing a rat in the starting chamber, located at the center of the platform, for $30 \mathrm{~s}$; the chamber is then raised, the aversive stimuli (bright light and high pitch noise) are switched on, and the rat is allowed to freely explore the maze for $120 \mathrm{~s}$. The purpose of ATs is to train the rats on finding the escape hole. A probe trial (PT) is similar to an AT except that the escape box has been removed, its purpose being to assess recent spatial memory retention. During PTs, the rats explore the maze for $90 \mathrm{~s}$. The behavioral performances were recorded using a computer-linked video camera mounted $110 \mathrm{~cm}$ above the platform. The performance of the subjects was determined using the Kinovea v0.7.6 (http://www.kinovea.org) software. The behavioral parameters assessed were as follows.

a. Escape box latency: Time (in s) spent by an animal since its release from the start chamber until it enters the escape box (during an AT) or until the first exploration of the escape hole (during a PT).

b. Nongoal hole exploration: Number of explorations of holes different from the escape one. Each exploration of an incorrect hole is counted as an error, provided that the rat lowers its nose below the plane of the table surface.

c. Exploration frequency: The times the rat explores every hole of the maze.

\section{Experimental Design}

The day for the first AT was termed experimental day 1. In a first stage, rats were submitted to the Barnes maze protocol for 9 days, each day involving four ATs. On the last day of this series, rats were submitted to a PT (PT0), $15 \mathrm{~min}$ after the last AT.

In order to follow the cognitive performance further, 1 month after PT0, the rats were submitted to a short reinforcement protocol of 3 days, of four ATs each. On the last day (Day 41), the subjects were submitted to another PT, the PT1 (Fig. 1B). The rationale for choosing this design was that replicating the test after 1 month would provide a more reliable estimate of spatial memory at each age than a single testing. At 


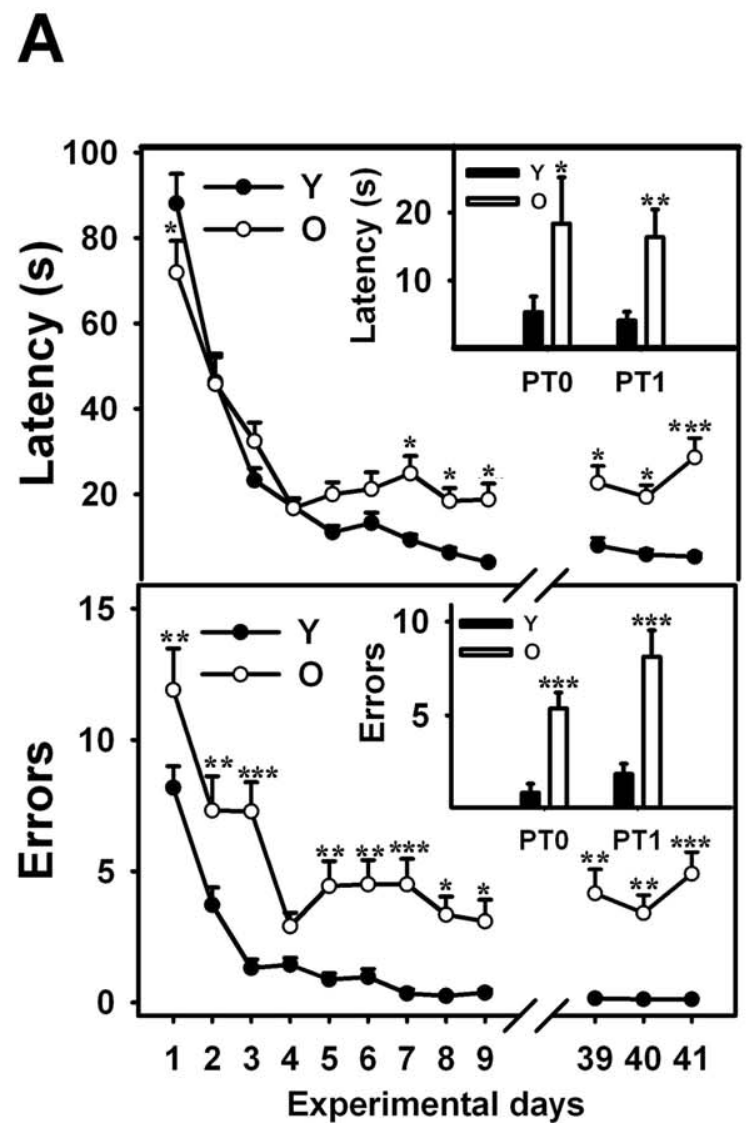

FIGURE 1. Effect of age on learning and spatial memory retention. Panel A, upper plot, shows escape hole latency throughout training and in probe trials (inset) in young and old rats. Panel A, lower plot, shows the number of errors during training and probe trials (inset) in the same animals. Panel B shows a diagram illustrating the experimental design used. Learning ability was assessed by performing 48 acquisition trials during 12 days (4 AT per day) with a 30-day interval between trial block 1-9 and trial

the young and old ages chosen, average spatial memory was expected to show little variation in a 30-day interval.

\section{Brain Processing}

At the end of the experiment, all rats were euthanized. Four rats from each group were sacrificed by rapid decapitation and their brains processed for transcriptomal analysis as described below. The remainder of the rats were placed under deep anesthesia and perfused with phosphate buffered para-formaldehyde $4 \%$, ( $\mathrm{pH} 7.4)$ fixative. The brains were removed and stored in para-formaldehyde $4 \%$, $(\mathrm{pH} 7.4)$ overnight at $4^{\circ} \mathrm{C}$. Brains were kept in cryopreservative solution at $-20^{\circ} \mathrm{C}$ until use. For immunohistochemical assessment, brains were cut coronally in $40-\mu \mathrm{m}$-thick sections with a vibratome (Leica).

\section{Immunohistochemistry}

All immunohistochemistry techniques were performed on free-floating sections. For each animal, separate sets of

B
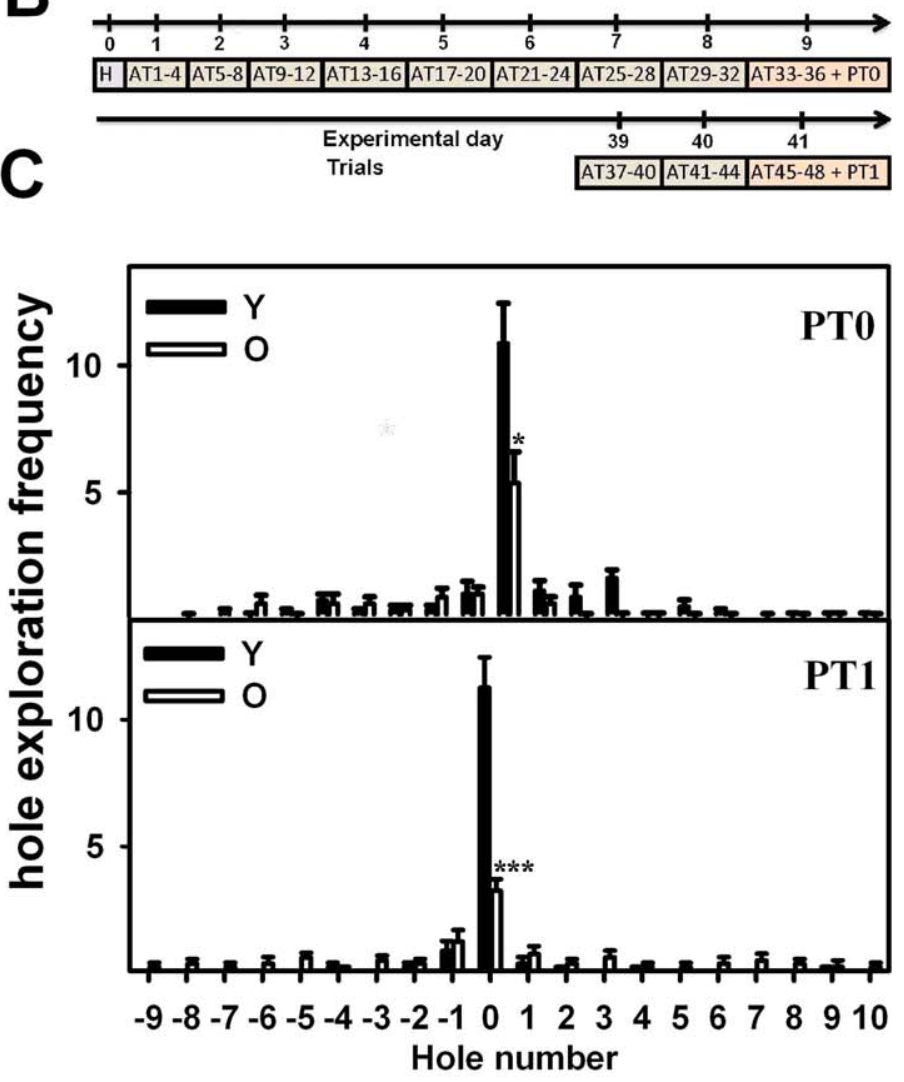

block 39-41. Panel C shows hole exploration frequency in probe trials 0 and 1 . Notice the sharp increase in exploratory frequency of hole \#0 (escape hole) and the age-related deterioration of frequency exploration at the escape hole. All data are represented as mean $\pm S E M$. Comparisons are made versus the corresponding young data point. ${ }^{*} P<0.05 ;{ }^{* *} P<0.01 ;{ }^{* *} P<0.001$. Number of young and old rats was 8 for every group. [Color figure can be viewed at wileyonlinelibrary.com]

sections were processed using either an anti-doublecortin goat polyclonal antibody (dilution 1:1000; Santa Cruz Biotechnology Cat\# sc-8066, RRID:AB_2088494) or an antiIba-1 rabbit polyclonal antibody (dilution 1:1000; Wako Cat\# 016-20001, RRID:AB_839506). Briefly, after overnight incubation at $4^{\circ} \mathrm{C}$ with the primary antibody, sections were incubated with biotinylated horse anti-goat serum (dilution 1:300; Vector Laboratories Cat\# BA-9500, RRID:AB_2336123), or a goat anti-rabbit serum (dilution 1:300; Vector Laboratories Cat\# BA-1000, RRID:AB_2313606), as appropriate, for $120 \mathrm{~min}$, rinsed and incubated with avidin-biotin-peroxidase complex (dilution 1:500; Vector Laboratories Cat\# PK-6100, RRID:AB_2336819) for 90 min and then incubated with 3, 3-diamino benzidinetretrahydro-chloride (DAB). Sections were counterstained with cresyl violet (Nissl staining) as described elsewhere (Morel et al., 2015). Finally, they were dehydrated and mounted with Vectamount (Vector) and used for image analysis. 


\section{Image Analysis}

In each hippocampal block, one every six serial sections was selected in order to obtain a set of noncontiguous serial sections spanning the dorsal hippocampus. Typically, a whole dorsal hippocampus comprises about 48 coronal sections, thus yielding eight sets of noncontiguous serial sections $(240 \mu \mathrm{m}$ apart). The number of cells was assessed in the dorsal hippocampus, which is typically located between coordinates -2.8 to $-4.5 \mathrm{~mm}$ relative to the bregma (Paxinos et al., 1998). For this task, we used an Olympus BX-51 microscope attached to an Olympus DP70 CCD video camera (Tokyo, Japan). All morphological parameters were assessed bilaterally.

Within the hippocampus, we focused our stereological assessment on immature neurons and microglial cells in the Dentate Gyrus (DG) and in the Stratum Radiatum (SR), respectively, two regions vulnerable to degeneration in $\mathrm{AD}$ patients (West et al., 1994).

\section{Neuroblast Analysis}

A stereological assessment of migrating neuroblasts, immunoreactive to doublecortin antibody (DCXir), was performed in the DG, including the subgranular zone (SGZ) and granular cell layer (GCL). DCXir cell number was assessed using a modified optical fractionator technique as previously described (Morel et al., 2015). To this end, the entire DG sections were used as the counting frame, making the area sampling fraction $($ asf $)=1$. The section sampling fraction (ssf) was $1 / 6$. Estimates were based on counting DCXir cell bodies as they came into focus. $N=3$ animals per group.

\section{Microglial Cell Analysis}

Microglial cells were identified as Iba-1 immunoreactive cells (Ibalir) and counted in the hippocampal SR, whose upper limit is the CA1 pyramidal layer, lower limit is Stratum Lacunosum Moleculare, and the lateral limit is the Stratum Lucidum of the dorsal hippocampus (Paxinos et al., 1998). To this end, a random grid consisting of squared probes (area $=5,625 \mu \mathrm{m}^{2}$ ) was superimposed over calibrated images taken at $\times 400 \mathrm{mag}$ nification and cells inside the probe area were counted. Cells making contact with boundary inclusion lines of the probes were included in the count, whereas cells in contact with boundary exclusion lines were not counted. Iba-1ir cells were morphologically classified as Type I, II, III, IV, and V on the basis of previously documented criteria (Diz-Chaves et al., 2012). Types I, II, and III were categorized as nonreactive glia whereas Types IV and V were taken as reactive glia. $N=3$ animals per group.

\section{RNA Extraction, Library Preparation, and Sequencing}

Four rats per group were euthanized and their right hippocampus dissected for transcriptome analysis. The hippocampus was stored at $-80^{\circ} \mathrm{C}$ until RNA extraction.
Tissues were homogenized in TRIzol Reagent (Life Technologies). The quality of the isolated RNA was assessed by measuring the RIN (RNA Integrity Number) using the Fragment Analyzer. Library preparation for RNA-Seq was performed using the truSeq RNA Sample Preparation Kit starting from 500 ng of total RNA. Accurate quantitation of cDNA libraries was performed using the QuantiFluor TM dsDNA System (Promega). The size range of final cDNA libraries was 280 $320 \mathrm{bp}$ and was determined applying the DNA Chip for NGS Libraries using the Fragment Analyzer (Advanced Analytical). cDNA libraries were amplified and sequenced by using the cBot and HiSeq2000 from Illumina (SR; 50 bp; ca. 30-35 million reads per sample). Raw datasets have been submitted to NCBI GEO database.

\section{Real-Time Quantitative PCR}

Total RNA was treated with gDNA wipeout and cDNA was synthesized with the Qiagen QuantiTect Reverse transcription kit (\#205310). qPCR was performed with the MESA BLUE qPCR MasterMix Plus for SYBR Assay Low ROX on a Stratagene Mx3000P qPCR system. The primers used are listed in Supporting Information Table S1.

\section{RNA-Seq Data Analysis}

Illumina HiSeq 2000 fluorescence images were transformed to BCL files with the Illumina BaseCaller software and samples were demultiplexed to FASTQ files with CASAVA (version 1.8.2). Sequencing quality was checked and approved via the FastQC software. Sequences were aligned to the genome reference sequence of Rattus norvegicus (RGSC assembly v5.0) using the STAR alignment software (Dobin et al., 2013; version 2.3.0e) allowing for two mismatches within 50 bases. Subsequently, resulting SAM files were converted to sorted BAM files, filtering of unique hits and counting was conducted with SAMtools (Li et al., 2009, version 0.1.18) and HTSeq (Anders et al., 2015; version 0.6.1p1).

To identify differentially expressed genes between hippocampal samples from young and old rats, we utilized the DESeq2 algorithm based on the normalized number of counts mapped to each gene (Love et al., 2014). Functional enrichment analyses were performed using the database for annotation, visualization and integrated discovery (DAVID, http://david.abcc. ncifcrf.gov/), Enrichr (http://amp.pharm.mssm.edu/Enrichr/), and InnateDB resource (http://www.innatedb.com/) resources based on the list of deregulated transcripts between hippocampal groups ( $P$-adj. $<0.05$; fold changes $>2$ ). Data integration and heatmap visualization of differentially expressed transcripts were done with $\mathrm{R}$ and the Multi Experiment Viewer software ( $\mathrm{MeV}$ v4.9) (Saeed et al., 2014). In addition, we used the ESTIMATE algorithm (Estimation of Stromal and Immune cells in Malignant Tumors using Expression data) to infer the immune component from each RNA-Seq Hippocampal sample (Yoshihara et al., 2013). 

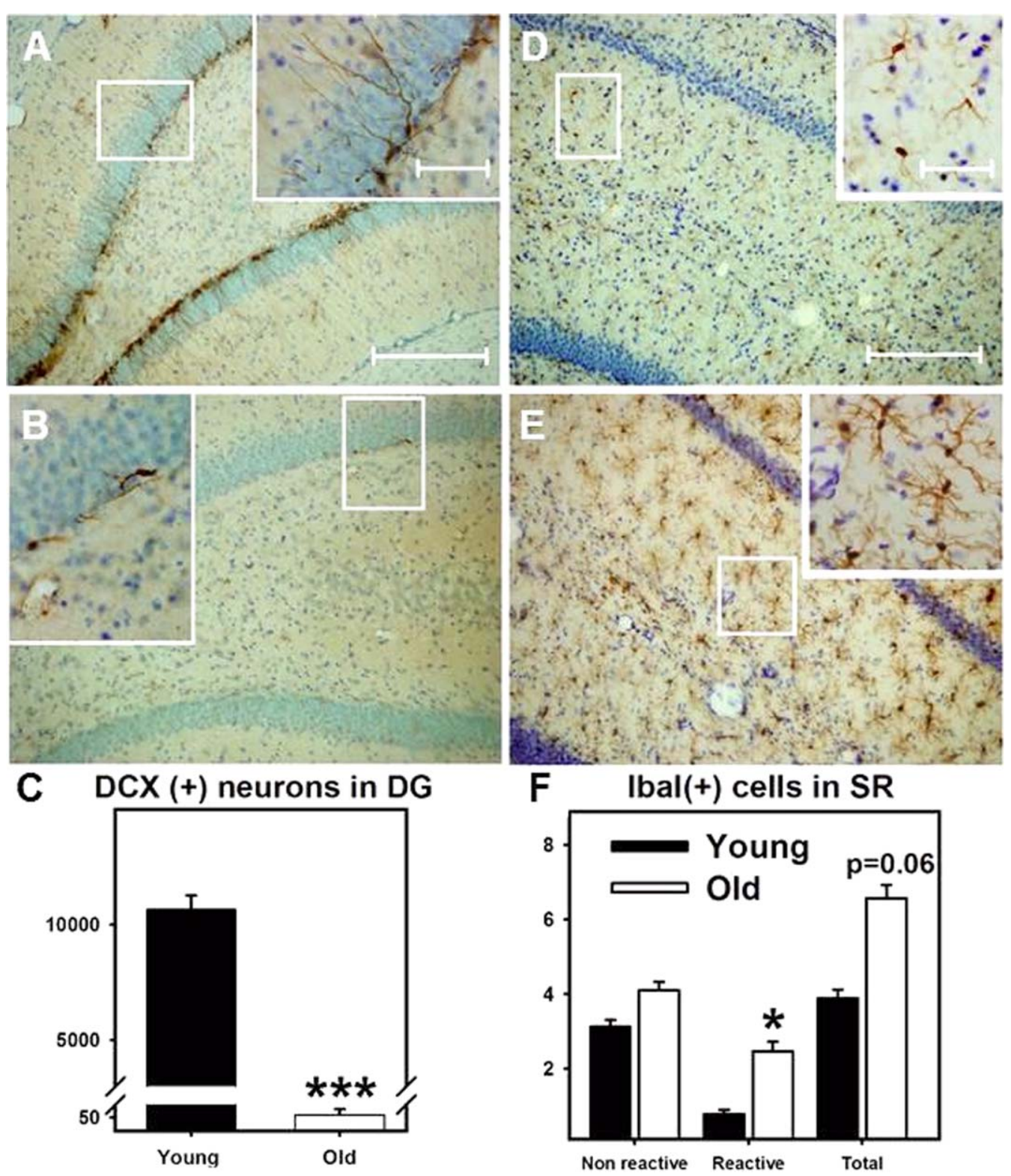

FIGURE 2. Doublecortin (DCX) and Iba-1 expression in the dorsal hippocampus of young and old rats. Images: Coronal sections of the dentate gyrus in representative animals of each age group showing DCXir cells (Panels A and B) and of the stratum radiatum for Iba-1 cells (Panels $D$ and $E$ ). DCX and Iba-1 cell numbers are plotted in Panel $C$ and F, respectively. Panels $A$ and

\section{Comparative Analysis of the Hippocampal Signature between Aging Rats and Human Counterparts}

Gene expression profile of deregulated genes $(n=210)$ obtained by RNAseq in the hippocampus of young and aging rats was assessed in a human context. Microarray data (Affymetrix) from normal human brain in aging individuals was obtained from the study GSE11882; CEL files were downloaded from GEO (http://www.ncbi.nlm.nih.gov/geo/),

D correspond to a representative young rat whereas panels $B$ and $E$ represent an old rat. Note the sharp age related fall in DCX cell numbers and the increase of reactive microglia (Iba- 1 ir cells) in the old rats. The number of hippocampi assessed was 3 for every group. Scale bars: $200 \mu \mathrm{m}$ for panoramic and $50 \mu \mathrm{m}$ for inset images. [Color figure can be viewed at wileyonlinelibrary.com]

processed and normalized with $\mathrm{R} /$ Bioconductor by using the fRMA package. The original dataset includes microarrays from four brain regions (hippocampus, entorhinal cortex, superior frontal gyrus, and postcentral gyrus) of cognitively intact humans (age 20-99 years). For the purpose of this study only hippocampal samples were considered $(n=43)$. From the 210 deregulated genes, a total of 170 orthologous genes were found in the human Affymetrix Platform (HGU-133 Plus_2), thus obtaining a final matrix of 43 samples vs. 170 genes. Samples were then categorized into two groups: young (age 20-50; 
A

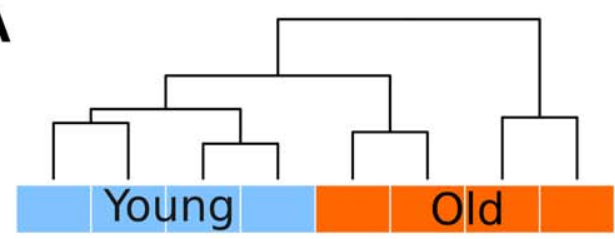

B

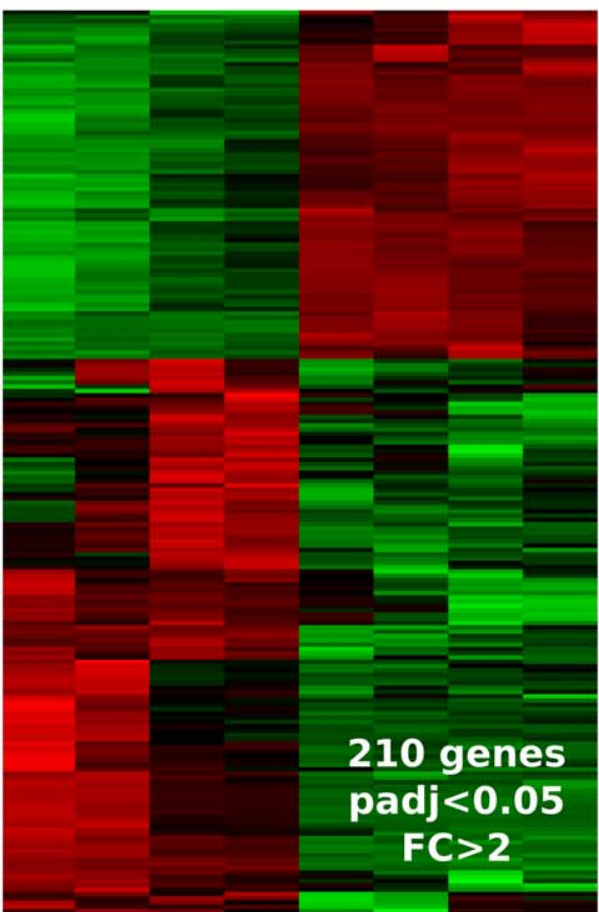

Up Down
C

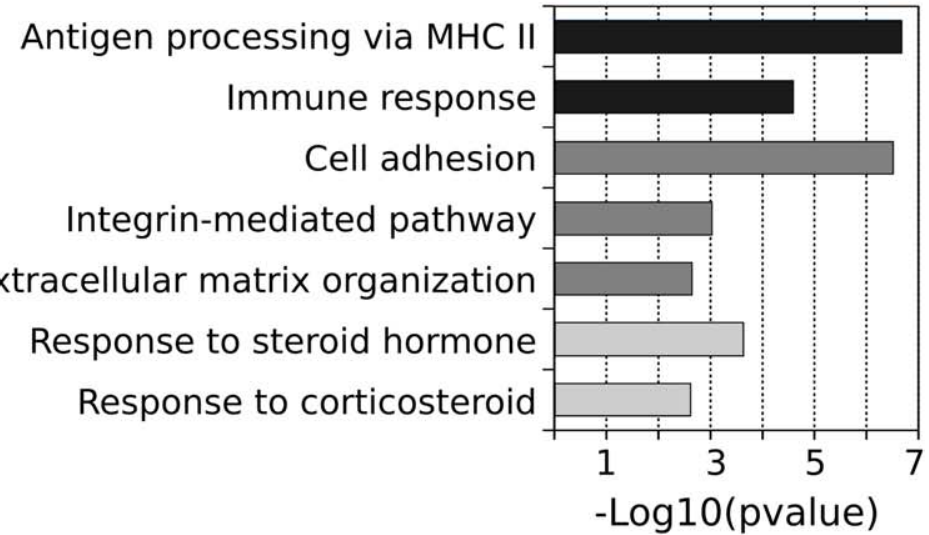

Hippocampus 5 vs $24 \mathrm{~m}$.

Entorhinal cortex 5 vs. 24 m.

Hippoc. CA3 region 6 vs. 25 m.

Hippocampus 3 vs $18 \mathrm{~m}$.

Spinal cord 6 vs. $18 \mathrm{~m}$.

Striatum 6 vs. $21 \mathrm{~m}$.

Rat

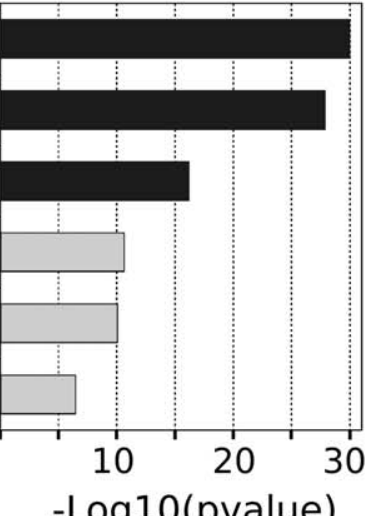

Mouse

-Log10(pvalue)
$\mathbf{E}$

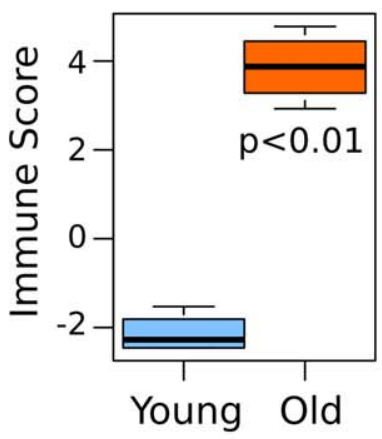

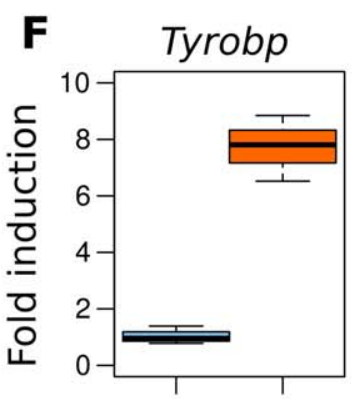

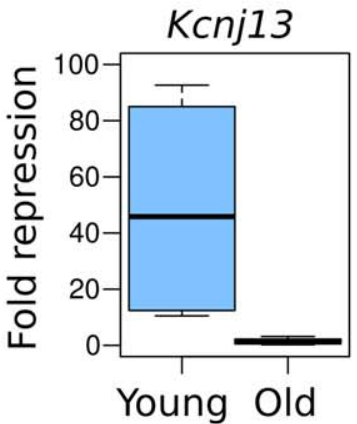

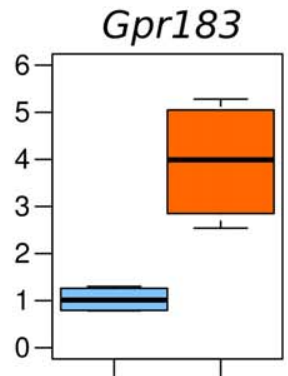
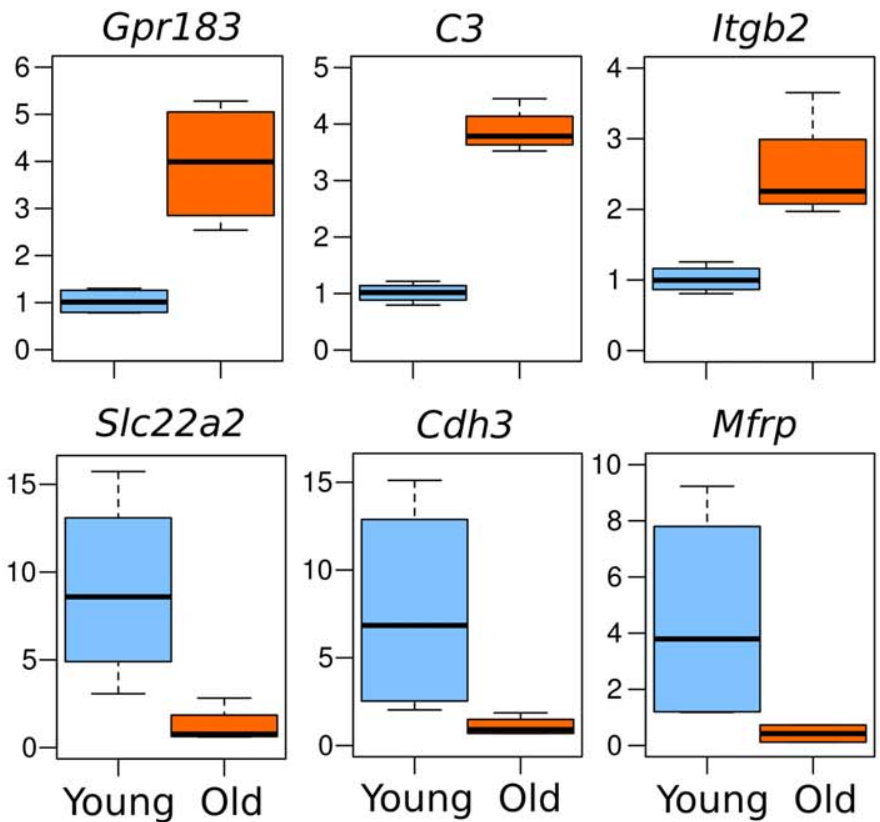

FIGURE 3. Age-related gene expression changes in the hippocampus. (A) Unsupervised hierarchical clustering of rat hippocampal samples. (B) Heat map of the 210 differentially expressed genes between young and old hippocampal samples. (C) Gene ontology (GO) analysis of deregulated transcripts revealed by functional enrichment analysis. (D) Set of genes commonly

deregulated across mice and rat models strongly associated with the aging process. (E) Box-plot depicting the immune score difference between young and old rat, as assessed with ESTIMATE. (F) Box-plot of eight representative genes validated by quantitative RT-PCR. The results confirm the RNA-seq data. [Color figure can be viewed at wileyonlinelibrary.com] 
$n=17$ ) and old (age 51-99; $n=26$ ). To get differentially expressed genes between the two groups, a $t$-test method was applied $(P<0.05)$.

\section{Statistical Analysis}

BM parameters and DCXir count estimates were analyzed with the Sigma Plot v.11 software (Systat Software, San Jose, CA). For BM latency and error analysis, the data from each day's ATs were averaged. These parameters were analyzed by two-way repeated measures ANOVA, considering group and day factors. When this ANOVA was significant, comparisons between means were performed with the Holm-Sidak post hoc test.

Statistics for Iba1-ir cell count was conducted with the Statistica 8 software (Tulsa, USA). Thus, we performed an analysis of variance (ANOVA) with a nested mixed model, in which variance was hierarchically decomposed into three categories, namely "Age group $\rightarrow$ Rat $\rightarrow$ Brain section." Age group was set as the fixed grouping variable, whereas rat and brain section were set as random variables. IbaI-ir count data were taken as dependent variable. When ANOVA was significant, post hoc LSD test for differences between means was performed. $P<0.05$ was set as significant.

In all cases, plots were performed with the Sigmaplot v.11 Software and show mean \pm SEM.

\section{RESULTS}

\section{Latency to Escape Box}

To assess hippocampal function in rats, we used a modified Barnes maze test. After Day 1, escape hole latency fell rapidly in young and old rats, reaching a plateau around experimental day 4 [two-way repeated measures (RM) ANOVA day factor degrees of freedom $(\mathrm{DF})=11 ; F=49.057 ; P<0.001]$. The plateau remained relatively constant for both age groups from Day 4 until Day 41 (the last day tested), a time span where latency remained consistently lower in the young as compared to the old rats (Fig. 1A, upper panel) (two-way RM ANOVA age factor $F=4.922 ; P=0.044$; Holm-Sidak test $P<0.05$ for Days $7,8,9,39,40$, and 41 ). As expected, the young group had a lower latency in both PT0 and PT1 (Mann-Whitney test $P=0.015$ and $P=0.003$ for PT0 and PT1, respectively) (inset in Fig. 1A, upper panel).

\section{Errors to Escape Box}

During the training series, the error number fell sharply in both age groups until reaching a plateau at Day 3-4 (two-way RM ANOVA day factor DF $=11 ; F=20.570 ; P<0.001)$. The young animals made significantly fewer errors than the old group on every experimental day, except for Day 4 (Fig. 1A, lower panel) (two-way RM ANOVA group factor $\mathrm{DF}=1$; $F=32.740 ; \quad P<0.001) \quad($ Holm-Sidak test for Day 4
$P=0.223)$. The young group made fewer errors than the old group in both PT0 and PT1 (Student's $t$-test $P<0.001$ and Mann-Whitney test $P<0.001$ for PT0 and PT1, respectively) (inset in Fig. 1A, lower panel).

\section{Hole Exploration Frequency}

In both age groups, hole exploration frequency showed a clear peak at hole 0 in both PT0 and PT1 (Fig. 1C). The amplitude of the peak was significantly higher in the young than in the old animals in both PTs (Student's $t$-test $P=0.015$ and Mann-Whitney test $P=<0.001$ for PT0 and PT1, respectively).

\section{Age-Associated Morphological Changes in the Dorsal Hippocampus}

In order to determine whether the behavioral alterations were associated with morphological changes in the brain, a stereological assessment of immature neurons and microglial cells was performed in the dorsal hippocampus of the young and aged rats. As expected, there was a sharp reduction in the number of DCXir[[strike_start]] [[strike_end]]cells, (immature neurons) in the DG of old rats as compared with their young counterparts (Student's $t$-test $P<0.001$ ) (Figs. 2A-C).

Hippocampal Ibalir microglial cells were classified as nonreactive and reactive, and were counted in the hippocampal SR. Although we found no significant differences in the number of non-reactive microglial cells (ANOVA factor group $\mathrm{F}=1.710 ; \mathrm{P}=0.262$ ) we observed a significant increase in reactive microglial cells in the old versus the young group (ANOVA group factor $\mathrm{F}=9.200 ; \mathrm{P}=0.0402$ ) (LSD test 0.0321). The total number of microglial cells (non-reactive and reactive) in the SR showed a trend towards an increase in the old versus young animals (ANOVA group factor $\mathrm{F}=5.175$; $\mathrm{P}=0.0624)$ (Figs. 2D-F).

\section{Age-Related Gene Expression Changes in the Hippocampus}

Next, we interrogated the transcriptome in order to identify gene expression differences that could underlie the morphological differences and explain the functional alterations observed. Unsupervised hierarchical clustering analysis of RNA-Seq profiles demonstrates a clear segregation of young and old hippocampal tissue (Fig. 3A). Statistical analysis of RNA-Seq data revealed 210 transcripts differentially expressed between young and old hippocampal samples ( $\mathrm{p}$-adj $<0.05$; Fold changes $>2$ ). Among the deregulated genes, 81 were up-regulated and 129 were down-regulated transcripts in old rats (Fig. 3B). The top 20 up- and down-regulated genes are shown in Table 1. This Table also shows the cell type in which the genes enlisted are commonly expressed. A complete list of deregulated genes is presented in Supporting Information File 1.

To identify biological processes that differentiate samples from old vs. young hippocampi, we performed functional enrichment analysis using DAVID. Gene ontology (GO) 
TABLE 1.

List of the Top 40 Deregulated (Up and Down) Hipoccampal Genes in Old Rats. Orange cells represent upregulated genes in old rats whereas green cells represent downregulated genes. RNA cell source has been obtained from public information on brain transcriptomics http://web. stanford.edu/group/barres_lab/brain_rnaseq.html. OPC, oligodendrial progenitor cell; NFO, newly formed oligodendrocyte; MO, myelinating oligodendrocyte. [Color table can be viewed at wileyonlinelibrary.com]

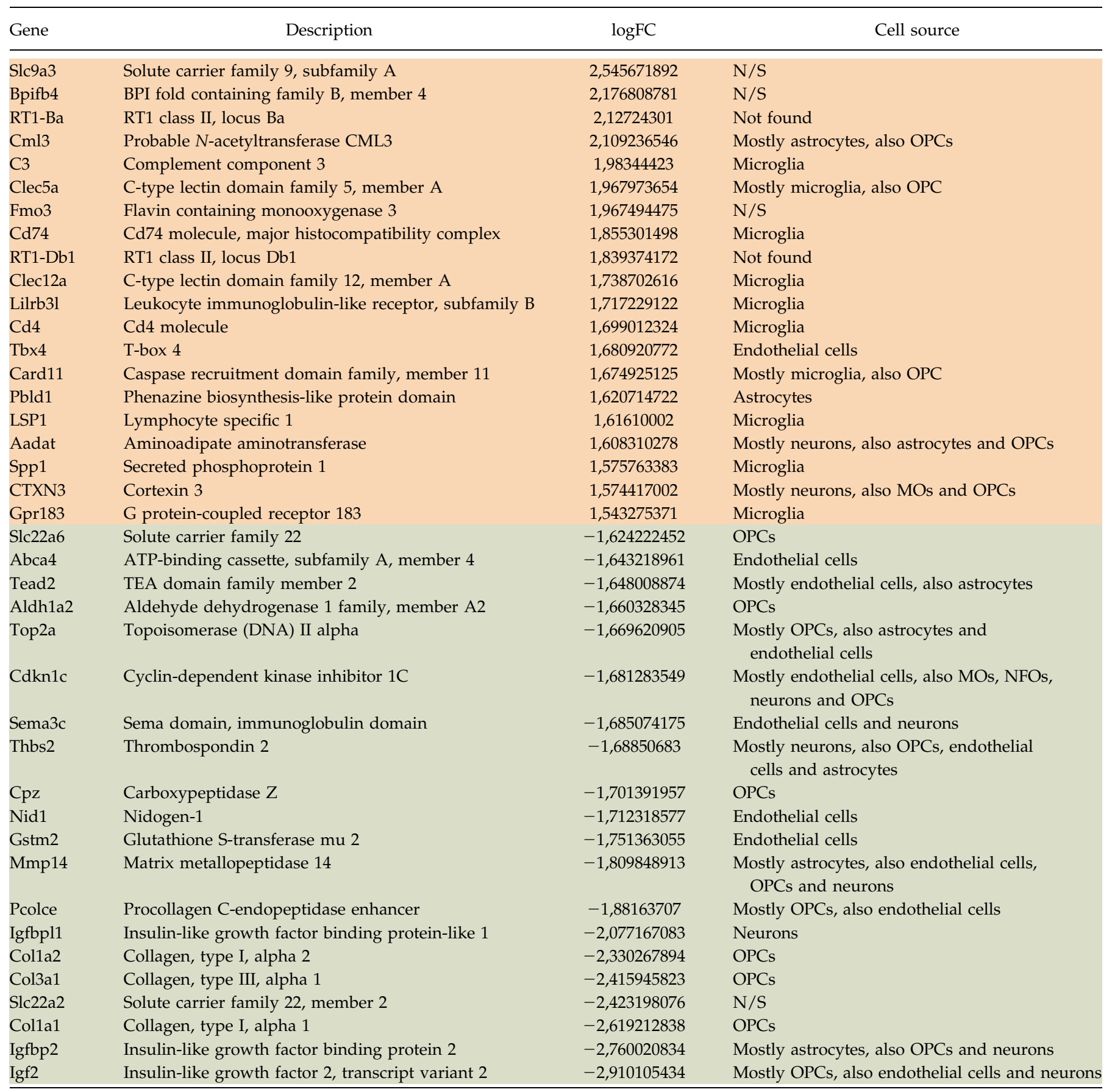

analysis of deregulated transcripts between the two groups of samples revealed specific GO terms related to antigen processing via MHC II- immune response, cell adhesion - integrinmediated pathway and response to corticosteroid - steroid hormones (Fig. 3C).
To identify commonly deregulated genes among our study and those identified in other age-related studies in animals, we performed an enrichment analysis from the 210 deregulated genes with an aging perturbation based database available at Enrichr online resource. This approach enabled us to identify a 
A
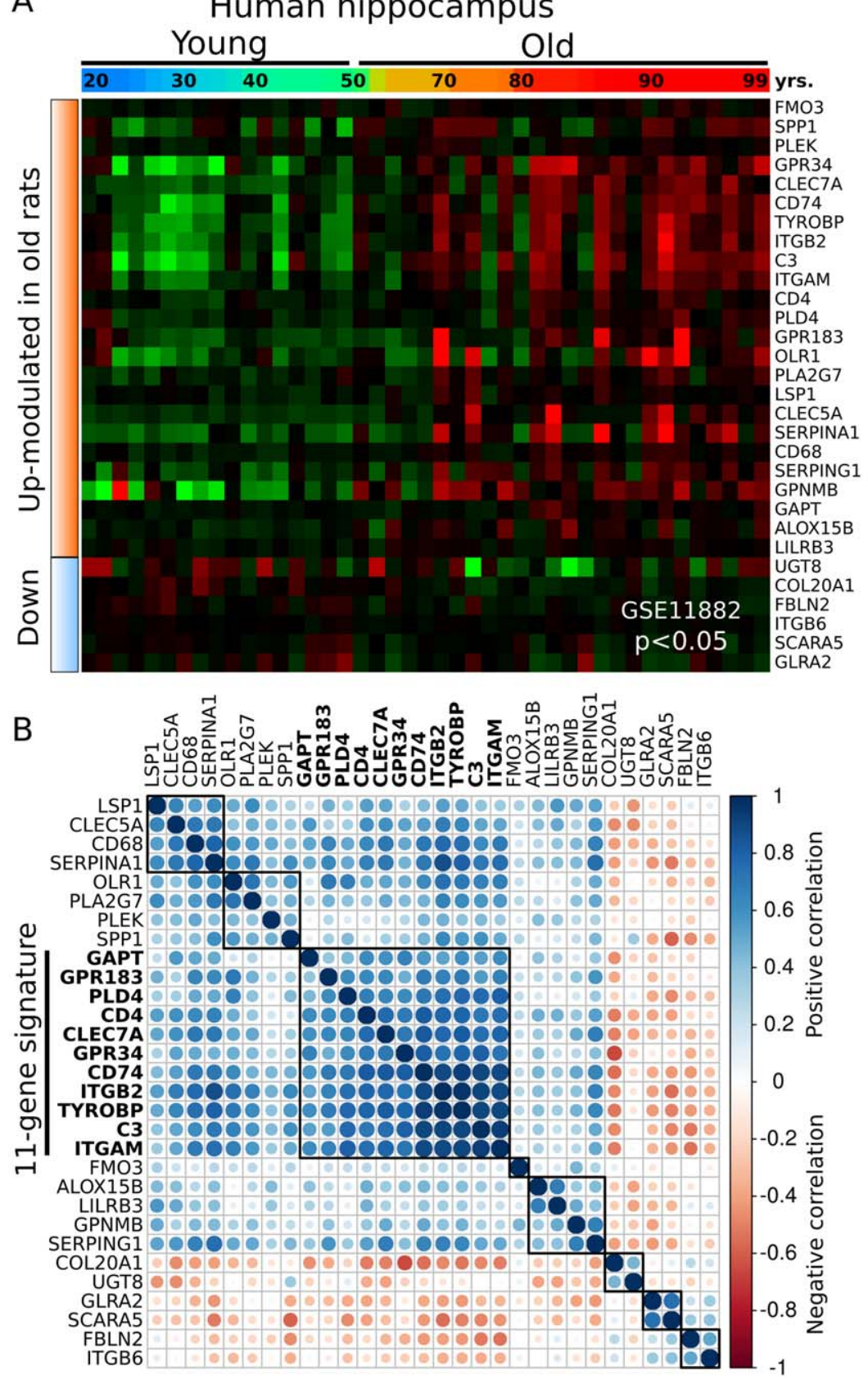

C
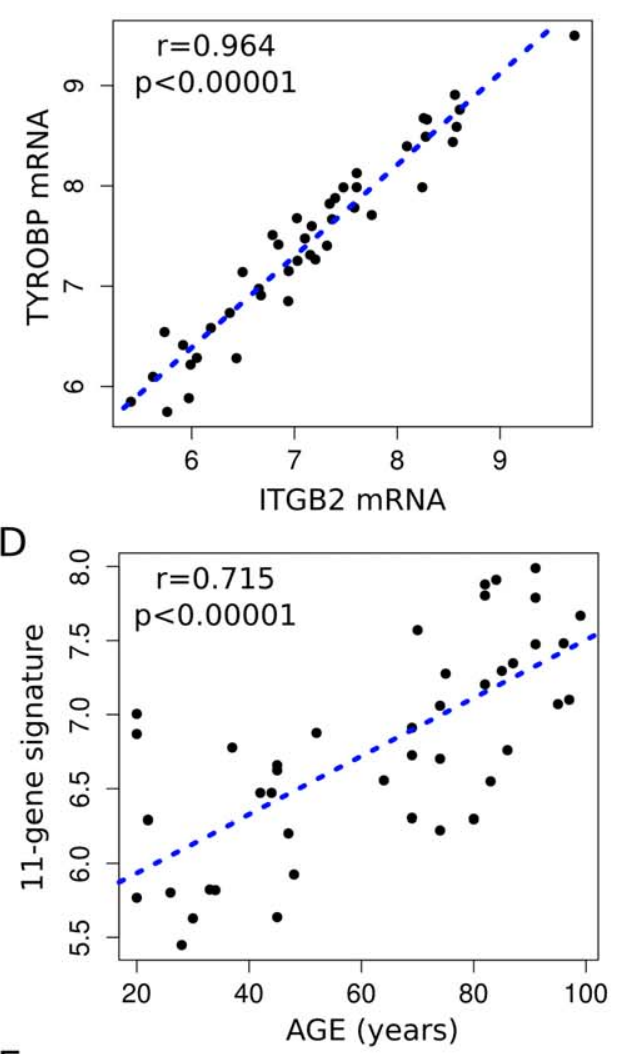

E

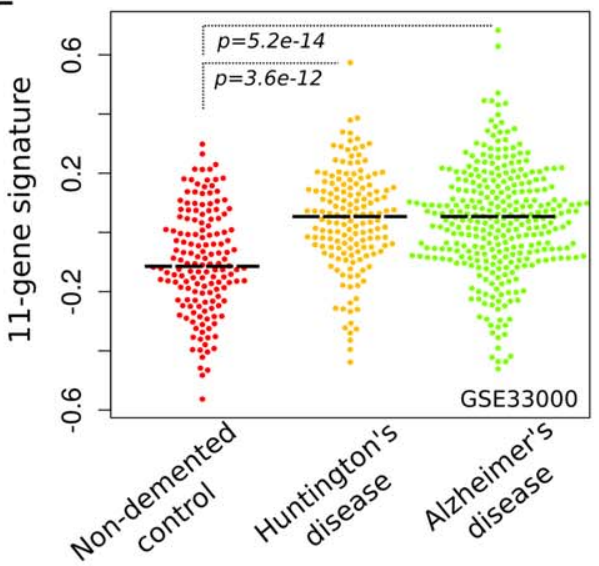

FIGURE 4. Hippocampal 11 gene-signature of deregulated genes conserved among species during aging (A) Heat map of the $30 \mathrm{DE}$ genes with aging in human and rat hippocampus. (B) Correlation matrix of the 30 deregulated genes, note the cluster of 11 genes showing the highest degree of correlation (11-gene signature). Dark blue intensity denotes high expression whereas circle diameter is proportional to the correlation value between the pair of genes in turn. (C) Expression correlation between tyrobp and itgb2 in human hippocampi. (D) Expression correlation of the 11 gene-signature related to aging in human hippocampi. (E) Expression of the 11 gene-signature in human hippocampi from old human individuals. [Color figure can be viewed at wileyonlinelibrary.com] set of genes associated with the aging process, significantly deregulated across male mice and male rat models (Fig. 3D, Supporting Information File 2).

Given the aforementioned association with immune-related processes, we developed a gene expression signature-based algorithm to predict the immune component among hippocampal samples. Interestingly, ESTIMATE predicted higher immune scores on old hippocampal samples compared with young brain samples $(P<0.01$, Fig. 3E).

In order to validate our RNA-Seq data we performed quantitative RT-PCR analysis of eight representative transcripts (Fig. $3 \mathrm{~F})$. Genes such as Tyrobp, C3, Itgb2, or gpr183, displayed an 
TABLE 2.

Description of the 11-Gene Signature

\begin{tabular}{|c|c|c|c|}
\hline Ensembl_Gene_ID & Gene symbol & Description & $\log F C$ \\
\hline ENSRNOG00000046834 & $\mathrm{C} 3$ & Complement component 3 & 1.98344423 \\
\hline ENSRNOG00000018735 & $\mathrm{Cd} 74$ & $\begin{array}{l}\text { Cd74 molecule, major histocompatibility complex, } \\
\text { class II invariant chain }\end{array}$ & 1.855301498 \\
\hline ENSRNOG00000016294 & $\mathrm{Cd} 4$ & Cd4 molecule & 1.699012324 \\
\hline ENSRNOG00000025094 & Gpr183 & G protein-coupled receptor 183 & 1.543275371 \\
\hline ENSRNOG00000033879 & Clec7a & C-type lectin domain family 7 , member A & 1.232456971 \\
\hline ENSRNOG00000039759 & Gpr34 & G protein-coupled receptor 34 & 1.222903325 \\
\hline ENSRNOG00000019728 & Itgam & Integrin, alpha $\mathrm{M}$ & 1.197102675 \\
\hline ENSRNOG00000020845 & Tyrobp & Tyro protein tyrosine kinase binding protein & 1.187167324 \\
\hline ENSRNOG00000026989 & Gapt & Grb2-binding adaptor protein, transmembrane & 1.17510715 \\
\hline ENSRNOG00000001224 & Itgb2 & Integrin, beta 2 (Itgb2) & 1.044682232 \\
\hline ENSRNOG00000028566 & Pld4 & Phospholipase D family & 1.030845136 \\
\hline
\end{tabular}

induction of 3- to 8-fold increase in the old animals, whereas Kcnj13, Cdh3, Mfrp, Scl22a2 behaved in the opposite way, with a 4- to 40-fold repression in old animals (Fig. 3F).

To further explore the differentially expressed genes found in rat hippocampus across aging, an in silico analysis in human hippocampal samples was performed. The comparison between the young and old groups yielded 30 deregulated genes among the 210 genes analyzed $(P<0.05)$, of which 24 were upregulated and 6 down-regulated in the group named old (Fig. 4A, Supporting Information File 2). The most prominent change was evidenced towards the 7 th decade, with a strong activation of genes such as CLEC7, TYROBP, CD74, ITGB2, C3, ITGAM, GPR183 and GAPT. Additionally, we computed a correlation matrix with the 30 deregulated genes and found that a cluster of 11 genes showed the highest degree of correlation. We named this cluster the 11-gene signature (Figs. 4B,C; Table 2). Next, we evaluated the 11-gene signature with respect to sex and found that 7 out of the 11 genes are significantly upregulated in the group "old" of both sexes, implying that the gene signature is independent of sex. Functional analysis of the 11-gene signature indicated an enrichment in processes related with the activation of immune response and MHC protein binding. These were the ontology terms more strongly enriched in the 210 deregulated genes identified in our animals, confirming the robustness and reproducibility of these 11 genes across species. Moreover, in the category of aging enrichment, 6 out of the 11-gene signature are significantly associated with the signature of the CA3 region of the old male rat hippocampus $(P<0.001$, GSE14724). The performance of the 11 -gene signature was also evaluated with respect to age and neurodegenerative conditions, such as Huntington's and Alzheimer's diseases. In both situations, a significant positive association was obtained (Figs. 4D,E; $P<0.001$ ).

We also evaluated the performance of the 11-gene signature on a dataset of Low Grade Brain Tumors obtained from the Cancer Genome Atlas (TCGA) $(\mathrm{n}=530)$, which includes astrocytomas, oligoastrocyomas and olidendrogliomas. The set of samples was clustered into two groups (named high and low) according to the expression of the 11-gene signature. A significant association between astrocytomas and the group high and oligodendrogliomas and the group low was observed $(P<0.01$; Supporting Information Fig. 1). A survival analysis was then performed which revealed that the group of samples belonging to the group high, mostly astrocytomas, had worse prognosis in overall survival and disease free survival $(P<0.01$; Supporting Information Fig. 1).

Finally, a protein-protein interaction network-based analysis employing STRING online resource indicated strong interactions among 7 out of 11 members of the 11-gene signature in rats. More importantly, protein-protein interactions between C3, Itgb2, Itgam, and Tyrobp were also conserved between rat and human species (Fig. 4C), suggesting that they are involved in an evolutionarily conserved bioprocess (Fig. 5).

\section{DISCUSSION}

\section{Functional Changes in the Aging Brain}

In rats, two important age-related changes take place in the brain during aging. At the functional level, there is an impairment in spatial memory performance as revealed by various tests of spatial learning and memory such as the radial arm maze (de Toledo-Morrell et al., 1984), the Morris Water Maze (MWM; Morris, 1984), and the Barnes maze (Barnes, 1979; Morel et al., 2015). This functional decline occurs in parallel with a marked reduction in hippocampal neurogenesis in aged rats (Barnes, 1979; Morel et al., 2015). Our results confirm that like in males, aging in female rats is associated with a decline in spatial memory performance as assessed by the Barnes maze, a less physically demanding test than the MWM, which is important as animals age. Our data also confirmed that in the hippocampal DG, aging brings about a severe fall in the number of immature neurons, a change that is likely to be causally involved in the decline of certain memory functions 


\section{Rattus norvegicus}

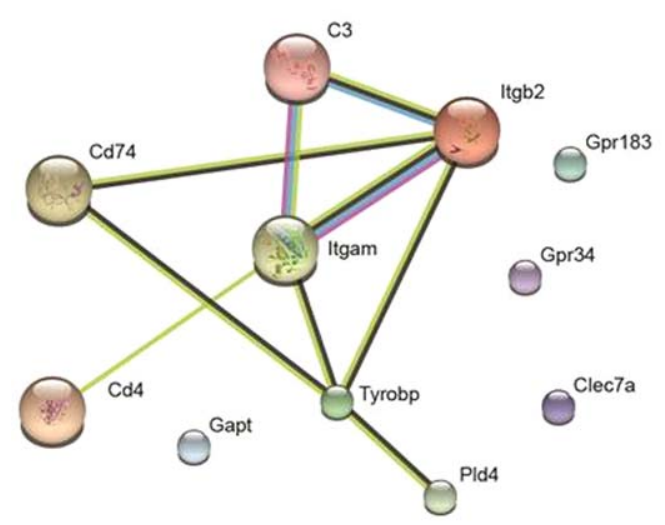

\begin{tabular}{|c|c|c|}
\hline \multicolumn{3}{|c|}{ Rattus norvegicus } \\
\hline node1 & $\underline{\text { node2 }}$ & $\underline{\text { score }}$ \\
\hline \multirow{2}{*}{ Itgam } & Itgb2 & 0.988 \\
\cline { 2 - 3 } & C3 & 0.976 \\
\hline \multirow{2}{*}{ Itgb2 } & C3 & 0.931 \\
\cline { 2 - 3 } & Tyrobp & 0.825 \\
\hline \multirow{2}{*}{ Itgam } & Cd4 & 0.692 \\
\cline { 2 - 3 } & Tyrobp & 0.683 \\
\hline \multirow{2}{*}{ Itgb2 } & Cd74 & 0.446 \\
\hline \multirow{2}{*}{ Tyrobp } & Pld4 & 0.423 \\
\cline { 2 - 3 } & Cd74 & 0.400 \\
\hline
\end{tabular}

FIGURE 5. Biological interactions of the 11-gene signature members. String 10.0 bioinformatics software (http://stringdb.org) was used to search for protein-protein interactions with the 11gene signature members (left: rat, right: human) in public databases and for the schematic representation of the network of functional interactions. Small nodes indicate protein of unknown 3D

in old rats (Yau et al., 2015). Some authors divide aging rats in age-impaired and age-unimpaired. We preferred not to use such a subdivision as we wanted to describe typical age-changes. Almost at every level, aging is known to lead to data scattering, be it serum hormone levels, circadian rhythms, etc. As expected, this also happened with some, but not all, cognitive parameters in young versus old rats and this led to a higher variance in the old than in the young animals. Had we subdivided the aging group, we would have been unable to tell which of the two groups represented typical age-related changes, marked impairment or lack/very mild impairment. In fact in our old rats we did not observe a spontaneous segregation into two groups. Rather, we observed a continuum from the less impaired old rats through the more impaired ones. Any subdivision would have to be based on an arbitrarily chosen limit.

Immune reactivity and inflammatory processes increase with aging in the brain (Lucin et al., 2009), with microglia playing a central role in this immune dysregulation (Luo et al., 2010; Norden et al., 2013). Innate immunity within the central
Homo sapiens

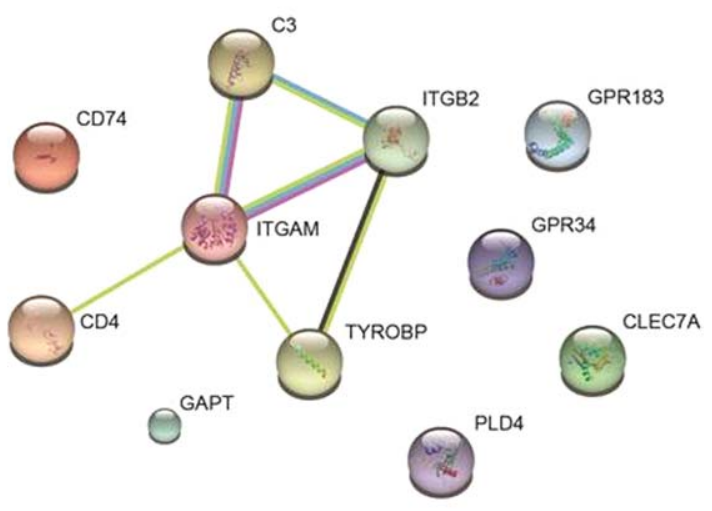

\begin{tabular}{|c|c|c|}
\hline \multicolumn{3}{|c|}{ Homo sapiens } \\
\hline node1 & $\underline{\text { node2 }}$ & $\underline{\text { score }}$ \\
\hline \multirow{2}{*}{ ITGAM } & C3 & 0.997 \\
\cline { 2 - 3 } & ITGB2 & 0.989 \\
\hline ITGB2 & C3 & 0.948 \\
\hline ITGAM & CD4 & 0.732 \\
\hline \multirow{2}{*}{ TYROBP } & ITGAM & 0.520 \\
\cline { 2 - 3 } & ITGB2 & 0.411 \\
\hline
\end{tabular}

structure, while large nodes indicate that some $3 \mathrm{D}$ structure is known or predicted. In STRING, each protein-protein interaction is annotated with one or more scores. These scores are indicators of confidence. All scores rank from 0 to 1 , being 1 the highest possible confidence. [Color figure can be viewed at wileyonlinelibrary.com]

nervous system is primarily provided by resident microglia, brain cells that are essential in immune surveillance and that also mediate the coordinated responses between the immune system and the brain. With normal aging, microglia develop a more reactive phenotype. For instance, in several models of aging there are increased levels of proinflammatory cytokines in the brain and increased expression of inflammation-associated receptors on microglia (Luo et al., 2010). There are also major differences in microglial biology between young and old age when the immune system is challenged and microglia activated. In this context, microglial activation is amplified and prolonged in the aged brain compared to adults. Prolonged microglial activation leads to the release of pro-inflammatory cytokines that exacerbate neuroinflammation, contributing to neuronal loss and impairment of cognitive function (Streit et al., 2004; Perry et al., 2010; Jiang et al., 2012). Our data are consistent with these alterations and demonstrate that in aging female rats there is a significant activation of reactive microglia in the hippocampus. 


\section{Gene-Expression Changes in the Hippocampus during Aging}

In male rodents, aging and, to a lesser extent, deficits in memory performance, have been associated with changes in hippocampal gene expression (Blalock et al., 2003; Verbitsky et al., 2004; Rowe et al., 2007; Burger et al., 2008). These differences consist mostly of gene upregulation in middleaged male mice (15-month old) as compared with 2-monthold counterparts (Verbitsky et al., 2004). In the CA1 hippocampal region of old male rats, 233 genes were found to be differentially expresssed with aging, $60 \%$ upregulated and $40 \%$ downregulated (Blalock et al., 2003). In the hippocampi from our female rats, $61 \%$ of the 210 transcripts differentially expressed in the old animals were downregulated suggesting that, in the hippocampus of very old animals, gene downregulation prevails. Therefore, a comparison between our females and old male rodents seems to point to a differential impact of age in males versus females concerning the proportion of hippocampal genes up and downregulated. Although this trend needs to be confirmed by further studies, a sex-related impact of age on the hippocampal transcriptome would, in general terms, be in line with the results of Sanguino et al. (2006) in rats and the observations reported in humans (Berchtold et al., 2008).

Our enrichment analysis from the 210 deregulated genes with aging revealed that there is a set of genes commonly deregulated during aging in male mice and rats. In aged male mice, the hippocampus was the brain region with the largest number of deregulated genes that were also present in the set of 210 deregulated genes we found in female rats.

As stated above, we and others have shown that one of most conspicuous changes associated with aging in the rat hippocampus is a sharp decline of neurogenesis in the hippocampal DG (Morel et al., 2015). It is not clear to us why such a substantial phenotypic change was not reflected by a significant deregulation of genes related with neurogenesis. It is likely that if the $N$ of the hippocampal samples analyzed had been higher the changes could have become significant. Neurogenesis is restricted to the DG; therefore, had we performed RNAseq from solely the DG region, it is likely that significant differences in the expression of these genes had become detectable.

Nevertheless, we want to point out that, in a recent study, we demonstrated that intracerebroventricular (ICV) IGF-I gene therapy increases the neurogenic activity in the DG of old rats (Pardo et al., 2016). Furthermore, in a follow-up study, yet unpublished, we found that ICV IGF-I gene therapy in the whole hippocampus of old rats significantly upregulates the expression of Sypl2, Nnat, and Itga-8, three genes related with synaptic processes. Expression of the neurogenesis-related gene DCX was also significantly upregulated by IGF-I gene therapy. Therefore, we expected a marked impact of aging on the expression of these genes. Since aging involves a constellation of gene expression changes, it could be possible that somehow, this prevented the deregulation of the above genes whereas, IGF-I therapy, which affected the expression of a much smaller number of genes, was able to significantly overexpress the above genes.

From the ESTIMATE-based score it is clear that, at least at the hippocampal level, aging in our female rats is strongly associated with a deregulation of a high number of immune genes, particularly those related to antigen processing via MHC II, immune response, cell adhesion and integrin-mediated pathways. The most affected glial element of the aged hippocampus seems to be the microglia where the deregulation of the TYROBP and CD11b genes may be pivotal to microglia dysfunction in the aged rats (Zhang et al., 2013).

\section{Hippocampal Aging- and Immune-Related Genes}

Our RNA-Seq data, validated by quantitative RT-PCR analysis of eight representative transcripts, show that various genes involved in the immune response, including TYROBP, CD11b, C3, CD18, CD4, and CD74, are overexpressed in aged female rats. Among them, TYROBP (tyrosine kinase binding protein) protein also known as DAP-12 (DNAX activating protein of $12 \mathrm{kDa}$ ) or KARAP (killer cell activating receptor associated protein) is a key regulator of the immune system. TYROBP acts as an adapter protein signaling numerous cell surface receptors, which play an important role in signal transduction in dendritic cells, osteoclasts, macrophages, and microglia. Accordingly, TYROBP serves as a hub of the "microglial sensome" network, on which major molecular connections are concentrated (Hickman et al., 2013). Loss of function in TYROBP gene causes Nasu-Hakola disease, which is characterized by progressive dementia and multifocal bone cysts (Xing et al., 2015). TYROBP is significantly upregulated in the brains of patients with Alzheimer's disease (Zhang et al., 2013). In the central nervous system TYROBP expression predominates in microglia, enhancing their phagocytic activity, which is responsible for clearing the $\beta$-amyloid deposits (Aß) and apoptotic neurons (Xing et al., 2015). TYROBP is also involved in the suppression of proinflammatory cytokine production responses by the microglia. Thus, TYROBP overexpression during aging may result in imbalances of the neuroinflammatory process.

Integrin CD11b (also known as Integrin alpha M, ITGAM) is one protein subunit that forms the heterodimeric integrin alpha-M beta- $2(\alpha \mathrm{M} \beta 2)$ molecule, also known as complement receptor 3 (CR3) (Ling et al., 2014) which positively regulates TLR4-induced signaling pathways in dendritic cells (Ling et al., 2014). In the brain, CD11b integrin expression is restricted to microglia and is a hallmark of their activation (Block et al., 2007). CD11b has recently been implicated in the death of dopaminergic neurons on inflammation (Pei et al., 2007). It has been demonstrated that CD11b and TYROBP (DAP12) are involved in the induction of developmental neuronal death in the hippocampus and that microglia use innate immunity mechanisms to induce the apoptosis of neurons committed to death during development (Wakselman et al., 2008). 
The relevance of the other immune genes that change with aging in the hippocampus is not obvious and will, therefore, not be further discussed here.

\section{A Conserved 11-Gene Signature Overexpressed during Aging in Rats and Humans}

An in silico analysis in human hippocampal samples performed to identify deregulated genes among the 210 genes analyzed in our rats, showed that from a group of 30 human hippocampal genes common to the deregulated female rat genes there is a cluster of 11 human genes showing the highest degree of correlation in a correlation matrix. When evaluated respective to age and neurodegenerative diseases, such as Huntington's and Alzheimer's, this 11-gene signature showed a significant positive association. Interestingly, a functional analysis of the 11-gene signature indicated that the deregulation of these genes is associated with an activation of immune response and MHC protein binding, which are the ontology terms more enriched by the 210 deregulated genes in the hippocampus of our aged female rats. The implication of this finding is that these 11 genes are deregulated in the aging hippocampus in rodents and humans and, since they are associated with an immune activation, it seems plausible to hypothesize that the activation of this 11-gene signature underlies a common dysfunctional mechanism of the immune system in the hippocampus during aging.

Some of the 11 signature genes have been discussed above. Others like Pld4, Gpr183, Glec7a, Gpr34, and Gapt will be briefly discussed here. Phospholipase D4 (Pld4) belongs to the Phospholipase D (PLD) family (Terao et al., 2013). Phospholipase $\mathrm{D}$ plays an important pathophysiological role in the progression of neurodegenerative diseases, primarily through its capacity as a signal transducer in indispensable cellular processes such as cytoskeletal reorganization and vesicle trafficking (Lindsley et al., 2012). Dysregulation of PLD by the protein $\alpha$-synuclein has been shown to lead to the specific loss of dopaminergic neurons in mammals. Disinhibition of PLD by $\alpha$-synuclein may contribute to Parkinson's deleterious phenotype (Peng et al., 2012). Abnormal PLD activity has also been suspected in Alzheimer's disease, where it has been observed to interact with presenilin 1 (PS-1), the principal component of the $\gamma$-secretase complex responsible for the enzymatic cleavage of amyloid precursor protein (APP).

G-protein coupled receptor 183 (GPR183,) also known as EBI2 (Epstein Barr-induced receptor 2) is a protein encoded by the GPR183 gene in humans (Rosenkilde et al., 2006). GPR183 helps B cell homing within a lymph node and its expression increases during B-cell activation, after B-cell receptor and CD40 stimulation (Pereira et al., 2009). C-type lectin domain family 7 member A (CLEC7A) belongs to a class of signaling pattern recognition receptors which are involved in antifungal immunity, but also play important roles in immune responses to other pathogens such as bacteria, viruses and nematodes (Drummond et al., 2011). Probable G-protein coupled receptor (GPR34) is a protein encoded by the GPR34 gene in humans (Schöneberg et al 1999). Growth factor receptorbound protein 2 (also known as Grb2 or Gapt) is an adaptor protein involved in signal transduction and cell communication. In humans, the GRB2 protein is encoded by the GRB2 gene (Matuoka et al., 1992; Lowenstein et al., 1992).

It will be interesting to determine whether the 11-gene signature is also deregulated during aging in other species as well as in other pathologies.

\section{Concluding Remarks}

The comprehensive characterization of age-related gene expression changes in the hippocampus allowed us to identify a group of 11 related genes commonly deregulated (overexpressed) across species during aging. The 11-gene signature may represent a cluster of immune and regulatory genes that are deregulated in the hippocampus and possibly other brain regions during aging as well as in some neurodegenerative diseases and low-grade brain tumors. This finding may offer the opportunity to seek suitable therapeutic targets among them, for the treatment of some brain pathologies.

\section{AUTHORS' CONTRIBUTIONS}

JP and GRM did the cognitive studies and the analysis of the behavioral data. JP and LF did the RT PCR analysis; MA, EL, TFO and RGG performed the analysis and interpretation of the RNA_seq data. JP, MA, EL and LF designed the different plots and graphs. JP, GRM, MA, EL, TFO, and RGG wrote different sections of the manuscript. GRM, TFO, and RGG assembled the final version of the paper.

\section{ACKNOWLEDGMENTS}

The authors thank to Mr. Thomas Lingner, MedicalUniversity Medical Center, Göttingen, for performing the transcriptome analysis, Yolanda Sosa for technical assistance and Oscar Vercellini and Araceli Bigres for animal care. MCA, EL, GRM, and RGG are career researchers of CONICET. TFO is supported by the DFG Center for Nanoscale Microscopy and Molecular Physiology of the Brain (CNMPB). JP is a recipient of a CONICET doctoral fellowship and was supported by a German-Argentine (Ale-Arg) travel fellowship.

The authors declare no conflict of interest.

\section{REFERENCES}

Anders S, Pyl PT, Huber W. 2015. HTSeq-A Python framework to work with high-throughput sequencing data. Bioinformatics 31: 166-169.

Barnes CA. 1979. Memory deficits associated with senescence: A neurophysiological and behavioral study in the rat. J Comp Physiol Psychol 93:74-104. 
Berchtold NC, Cribbs DH, Coleman PD, Rogers J, Head E, Kim R, Beach T, Miller C, Troncoso J, Trojanowski JQ, Zielke HR, Cotman CW. 2008. Gene expression changes in the course of normal brain aging are sexually dimorphic. Proc Natl Acad Sci U S A 105:15605-15610.

Blalock EM, Chen KC, Sharrow K, Herman JP, Porter NM, Foster TC, Landfield PW. 2003. Gene microarrays in hippocampal aging: Statistical profiling identifies novel processes correlated with cognitive impairment. J Neurosci 23:3807-3819.

Block ML, Zecca L, Hong JS. 2007. Microglia-mediated neurotoxicity: Uncovering the molecular mechanisms. Nature Rev Neurosci 8: 57-69.

Burger C, López MC, Feller JA, Baker HV, Muzyczka N, Mandel RJ. 2007. Changes in transcription within the CA1 field of the hippocampus are associated with age-related spatial learning impairments. Neurobiol Learn Mem 87:21-41.

Burger C, Lopez MC, Baker HV, Mandel RJ, Muzyczka N. 2008. Genome-wide analysis of aging and learning-related genes in the hippocampal dentate gyrus. Neurobiol Learn Mem 89:379-96.

de Toledo-Morrell L, Morrell F, Fleming S. 1984. Age-dependent deficits in spatial memory are related to impaired hippocampal kindling. Behav Neurosci 98:902-907.

Diz-Chaves Y, Pernía O, Carrero P, Garcia-Segura LM. 2012. Prenatal stress causes alterations in the morphology of microglia and the inflammatory response of the hippocampus of adult female mice. J Neuroinflam 9:71-80.

Dobin A, Davis CA, Schlesinger F, Drenkow J, Zaleski C, Jha S, Batut P, Chaisson M, Gingeras TR. 2013. STAR: Ultrafast universal RNA-seq aligner. Bioinformatics 29:15-21.

Drummond RA, Brown GD. 2011. The role of Dectin-1 in the host defence against fungal infections. Curr Opin Microbiol 14:392399.

Hickman SE, Kingery ND, Ohsumi TK, Borowsky ML, Wang LC, Means TK, El Khoury J. 2013. The microglial sensome revealed by direct RNA sequencing. Nat Neurosci 16:1896-1905.

Jiang T, Yu JT, Tan L. 2012. Novel disease-modifying therapies for Alzheimer's disease. J Alz Dis 31:475-492.

Li H, Handsaker B, Wysoker A, Fennell T, Ruan J, Homer N, Marth G, Abecasis G, Durbin R. 1000 Genome Project Data Processing Subgroup. 2009. The Sequence Alignment/Map format and SAMtools. Bioinformatics 25:2078-9. 15

Lindsley CW, Brown HA. 2012. Phospholipase D as a therapeutic target in brain disorders. Neuropsychopharm 37:301-302.

Ling GS, Bennett J, Woollard KJ, Szajna M, Fossati-Jimack L, Taylor PR, Scott D, Franzoso G, Cook HT, Botto M. 2014. Integrin CD11b positively regulates TLR4-induced signalling pathways in dendritic cells but not in macrophages. Nat Commun 5:30393050.

Love MI, Huber W, Anders S. 2014. Moderated estimation of fold change and dispersion for RNA-seq data with DESeq2. Genome Biol 15:550-570.

Lowenstein EJ, Daly RJ, Batzer AG, Li W, Margolis B, Lammers R, Ullrich A, Skolnik EY, Bar-Sagi D, Schlessinger J. 1992. The SH2 and $\mathrm{SH} 3$ domain-containing protein GRB2 links receptor tyrosine kinases to ras signaling. Cell 70:431-442.

Lucin KM, Wyss-Coray T. 2009. Immune activation in brain aging and neurodegeneration: Too much or too little? Neuron 64:110122.

Luo XG, Ding JQ, Chen SD. 2010. Microglia in the aging brain: Relevance to neurodegeneration. Mol Neurodeg 5:12- 20.

Matuoka K, Shibata M, Yamakawa A, Takenawa T. 1992. Cloning of ASH, a ubiquitous protein composed of one Src homology region (SH) 2 and two $\mathrm{SH} 3$ domains, from human and rat cDNA libraries. Proc Natl Acad Sci U S A 89:9015-9029.

Morel GR, Andersen T, Pardo J, Zuccolilli GO, Cambiaggi VL, Hereñú CB, Goya RG. 2015. Cognitive impairment and morphological changes in the dorsal hippocampus of very old female rats. Neuroscience 303:189-199.

Morris R. 1984. Developments of a water-maze procedure for studying spatial learning in the rat. J Neurosci Meth 11:47-60.

Norden DM, Godbout JP. Mi 2013. Neuropathol Appl Neurobiol 39: 19-34. Microglia of the aged brain: Primed to be activated and resistant to regulation.

O'Keefe J, Nadel L. 1978. The Hippocampus as a Cognitive Map. Claredon Press, Oxford, UK: Oxford University Press. ISBN 0-19857206-9.

Paxinos G, Watson C. 1998. 4th Ed. The Rat Brain in Stereotaxic Coordinates. San Diego, CA: Academic Press. ISBN 0-12-547619-1.

Pardo J, Uriarte M, Cónsole GM, Reggiani PC, Outeiro TF, Morel GR, Goya RG. 2016. Insulin-like growth factor-I gene therapy increases hippocampal neurogenesis, astrocyte branching and improves spatial memory in female aging rats. Eur J Neurosci Aug; 44:2120-8.

Pei Z, Pang H, Qian L, Yang S, Wang T, Zhang W, Wu X, Dallas S, Wilson B, Reece JM, Miller DS, Hong JS, Block M. 2007. MAC1 mediates LPS induced production of superoxide by microglia: The role of pattern recognition receptors in dopaminergic neurotoxicity. Glia 55:1362-1373.

Peng X, Frohman MA. 2012. Mammalian phospholipase D physiological and pathological roles. Acta Physiol 204:219-226.

Pereira JP, Kelly LM, Xu Y, Cyster JG. 2009. EBI2 mediates B cell segregation between the outer and centre follicle. Nature 460: $1122-1126$.

Perry VH, Nicoll JA, Holmes C. 2010. Microglia in neurodegenerative disease. Nat Rev Neurol 6:193-201.

Rosenkilde MM, Benned-Jensen T, Andersen H, Holst PJ, Kledal TN, Lüttichau HR, Larsen JK, Christensen JP, Schwartz TW. 2006. Molecular pharmacological phenotyping of EBI2: An orphan seven-transmembrane receptor with constitutive activity. J Biol Chem 281:13199-13208.

Rowe WB, Blalock EM, Chen KC, Kadish I, Wang D, Barrett JE, Thibault O, Porter NM, Rose GM, Landfield PW. 2007. Hippocampal expression analyses reveal selective association of immediate-early, neuroenergetic, and myelinogenic pathways with cognitive impairment in aged rats. J Neurosci 27:3098-3110.

Saeed AI, Sharov V, White J, Li J, Liang W, Bhagabati N, Braisted J, Klapa M, Currier T, Thiagarajan M, Sturn A, Snuffin M, Rezantsev A, Popov D, Ryltsov A, Kostukovich E, Borisovsky I, Liu Z, Vinsavich A, Trush V, Quackenbush J. 2003. TM4: A free, open-source system for microarray data management and analysis. Biotechniques 34:374-378.

Sanguino E, Roglans N, Rodríguez-Calvo R, Alegret M, Sánchez RM, Vázquez-Carrera M, Laguna JC. 2006. Ageing introduces a complex pattern of changes in several rat brain transcription factors depending on gender and anatomical localization. Exp Gerontol 41:372-379.

Schöneberg T, Schulz A, Grosse R, Schade R, Henklein P, Schultz G, Gudermann T. 1999. A novel subgroup of class I G-proteincoupled receptors. Biochim Biophys Acta 1446:57-70.

Squire LR, Knowlton B, Musen G. 1993. The structure and organization of memory. Annu Rev Psychol 44:453-495.

Streit WJ, Mrak RE, Griffin WS. 2004. Microglia and neuroinflammation: A pathological perspective. J Neuroinflammation 1:14-17.

Terao C, Ohmura K, Kawaguchi Y, Nishimoto T, Kawasaki A, Takehara K, Furukawa H, Kochi Y, Ota Y, Ikari K, Sato S, Tohma S, Yamada R, Yamamoto K, Kubo M, Yamanaka H, Kuwana M, Tsuchiya N, Matsuda F, Mimori T. 2013. PLD4 as a novel susceptibility gene for systemic sclerosis in a Japanese population. Arthr Rheum 65:472-480.

Verbitsky M, Yonan AL, Malleret G, Kandel ER, Gilliam TC, Pavlidis P. 2004. Altered hippocampal transcript profile accompanies an age-related spatial memory deficit in mice. Learn Mem 11:253260. 
Wakselman S, Béchade C, Roumier A, Bernard D, Triller A, Bessis A. 2008. Developmental neuronal death in hippocampus requires the microglial CD11b integrin and DAP12 immunoreceptor. J Neurosci 6: 28:8138-8143.

West M, Coleman P, Flood D, Troncoso J. 1994. Differences in the pattern of hippocampal neuronal loss in normal ageing and Alzheimer's disease. Lancet 344:769-772.

Xing J, Titus AR, Humphrey MB. 2015. The TREM2-DAP12 signaling pathway in Nasu-Hakola disease: A molecular genetics perspective. Res Rep Biochem 5:89-100.

Yau SY, Li A, So KF. 2015. Involvement of adult hippocampal neurogenesis in learning and forgetting. Neural Plast 5:1-13.

Yoshihara K, Shahmoradgoli M, Martínez E, Vegesna R, Kim H, Torres-Garcia W, Treviño V, Shen H, Laird PW, Levine DA,
Carter SL, Getz G, Stemke-Hale K, Mills GB, Verhaak RG. 2013. Inferring tumour purity and stromal and immune cell admixture from expression data. Nat Commun 4:2612-2622.

Zhang B, Gaiteri C, Bodea LG, Wang Z, McElwee J, Podtelezhnikov AA, Zhang C, Xie T, Tran L, Dobrin R, Fluder E, Clurman B, Melquist S, Narayanan M, Suver C, Shah H, Mahajan M, Gillis T, Mysore J, MacDonald ME, Lamb JR, Bennett DA, Molony C, Stone DJ, Gudnason V, Myers AJ, Schadt EE, Neumann H, Zhu J, Emilsson V. 2013. Integrated systems approach identifies genetic nodes and networks in late-onset Alzheimer's disease. Cell 153: 707-720.

http://www.ncbi.nlm.nih.gov/gene? $\mathrm{Db}=$ gene $\& \mathrm{Cmd}=$ ShowDetailView $\&$ TermToSearch=2857. Entrez Gene: GPR34 G protein-coupled receptor 34 . 